\title{
Competition and co-operation in coral colonies
}

\author{
ANDREW JAMES RiXON
}

This thesis considers an implicitly spatial model (Cellular Autornaton model) to investigate the effects of space on stability/coexistence, diversity, pattern formation and invisibility within community structures.

Landscape size is fundamental to the stability of the model, and to the species richness found in the equilibrium state. A discrete Markov model and a continuous differential equation model are used to compare the dynamical responses of homogeneous models with those of a heterogeneous cellular automaton model. The explicitly spatial heterogeneous model allows coexistence of four species over a large range of parameter spaces, whereas the homogeneous models have limiting dynamics resulting in three species coexistence.

Within the model, disturbance spread is damped to a 'tentacle-like' spread in large landscapes. This damping leads to a decrease in the total disturbance response, maintaining diversity.

Any form of pattern or spatial order predisposes the model to being highly sensitive to perturbation. The generation of spatial diversity is dependent on the initial spatial configuration; in disordered regions patterned structures are created, while highly ordered structures become disordered. This behaviour is monitored through an entropy mapping for the model. This mapping determines (for any spatial scenario) what the spatial equilibrium's response will be.

Finally, the model is used to explore the effects of connectivities of species interactions and of space on the success of invading species. Connectivity of the rule structures (species interactions) is found to be the major determinant in success or failure of establishment for an invading species. The effect of landscape size on invasion is secondary.

Received 15th May, 2000

Thesis submitted to The University of Queensland, September 1999. Degree approved, February 2000. Supervisors: Dr. Alan Jones and Professor Craig.

Copyright Clearance Centre, Inc. Serial-fee code: 0004-9727/00 \$A2.00+0.00. 
In summary, the thesis uses a simulation model to explore possible ecological theories and paradigms concerning the importance of space and spatial processes to ecological/biological phenomena.

Intelligenesis Corporation

50 Broadway

New York NY 10007

United States of America 